\title{
The accelerated observer with back-reaction effects
}

\author{
Roberto Casadio* and Giovanni Venturi ${ }^{\dagger}$ \\ Dipartimento di Fisica, Università di Bologna \\ and \\ Istituto Nazionale di Fisica Nucleare, Sezione di Bologna \\ via Irnerio 46, 40126 Bologna, Italy
}

July 30,2018

\begin{abstract}
The quantum mechanical evolution of an accelerated extended detector coupled to a massless scalar field is exhibited and the back-reaction due to emission or absorption processes computed at first order in the change of the detector's mass and acceleration. An analogy with black hole evaporation is found and illustrated.
\end{abstract}

PACS numbers: 03.65.Sq, 04.70.Dy

List of keywords:

Accelerated observer

Back-reaction

Black hole evaporation

Two field detector

Unruh effect

*e-mail: casadio@bo.infn.it

${ }^{\dagger}$ e-mail: armitage@bo.infn.it 


\section{Introduction}

Because of the equivalence principle field theory in the presence of gravitational fields is related to that in accelerated systems. Indeed, subsequently to Hawking's remarkable discovery that black holes behave as if they had an effective temperature of $\hbar / 8 \pi G m$ with $m$ the mass of the black hole and $G$ Newton's constant [1], it was found that a detector with uniform acceleration in the usual vacuum state of flat Minkowski space will be thermally excited to a temperature $T=\hbar a / 2 \pi[2]$.

It is therefore to be hoped that the study of the, apparently simpler, Unruh effect may shed light on the case of a curved space-time. In particular, in his original paper [2] Unruh suggested a two-field model for a finite mass accelerated detector which consisted of two scalars of differing mass. This corresponds to a detector of finite mass having two energy levels separated by a gap corresponding to the mass difference of the two fields. A point-like (infinite mass) monopole detector again having a finite energy internal degree of freedom was suggested, on the other hand, by DeWitt [3]. It is clear that, if one considers a finite mass for the detector, one must not ignore the quantum mechanical smearing of the trajectory or the recoil back-reaction when a quantum is emitted (absorbed). For the former reason we have previously [4] considered a massless neutral scalar field $\varphi$ coupled to a finite mass quantum monopole detector described by a gaussian wave-packet which was allowed to evolve according to an inverted harmonic oscillator potential, corresponding to constant acceleration. On examining the probability per unit time that the detector be excited by the absorption of scalar quanta, we observed that, on first considering the classical limit $(\hbar \rightarrow 0)$ and then the point-like limit for the gaussian wave-packet, we reproduced the usual results (Unruh effect [2]). If however one considered the point-like limit first, the detector decoupled from the scalar field. This rather surprising result is associated with the fact that, once quantum-mechanical evolution is considered, the Compton wavelength of the detector enters in the theory.

In our previous approach the scalar field only modified the internal degree of freedom and did not influence the motion or mass of the detector. The purpose of this letter is to include the back-reaction on the trajectory due to the emission (absorption) of massless scalar quanta (our motivation is an analogy with Bremsstrahlung wherein a charged particle decelerates by emitting soft photons and follows a trajectory of changing energy and acceleration). This will be done by modifying our previous approach in analogy with Unruh's two-field model in order to eliminate the need for the monopole moment (or internal degree of freedom) of the detector. We shall then describe a "detector" with changing acceleration and mass due to the emission (absorption) of scalar quanta which will then mimic black hole evaporation thus generalizing the original Unruh effect.

We use units such that $c$ and the Boltzmann constant are equal to one.

\section{Two field model}

To illustrate our approach it will be sufficient (and easier) to consider a 2-dimensional Minkowski space-time with coordinates $x^{0}, z$ (for respectively Minkowski time and space) and an effective Lagrangian density

$$
\mathcal{L}\left(z, x^{0}\right)=\int d \tau \sum_{i=1}^{2} \delta\left(x^{0}-x_{i}^{0}\right)
$$




$$
\begin{aligned}
& \times\left[i \hbar \psi_{i}^{*}(z, \tau) \dot{\psi}_{i}(z, \tau)+\frac{\hbar^{2}}{4 m_{i}}\left|\frac{\partial}{\partial z} \psi_{i}(z, \tau)\right|^{2}-m_{i} a_{i}^{2} z_{i}^{2}\left|\psi_{i}(z, \tau)\right|^{2}\right] \\
+ & \frac{1}{2} \int d \tau \delta\left(x^{0}-\frac{1}{2}\left(x_{1}^{0}+x_{2}^{0}\right)\right) Q\left[\psi_{2}^{*}(z, \tau) \psi_{1}(z, \tau)+\psi_{2}(z, \tau) \psi_{1}^{*}(z, \tau)\right] \varphi(z, \tau) \\
- & \frac{1}{2} \eta^{\mu \nu} \partial_{\mu} \varphi \partial_{\nu} \varphi
\end{aligned}
$$

where $x_{i}^{0}=a_{i}^{-1} \sinh a_{i} \tau, a_{i}$ (positive) is the proper acceleration in the $z$ direction and a dot denotes differentiation with respect to the continuous proper time $\tau$ which parametrizes the (semi)classical trajectory followed by the observer $\psi$. The diverse $\psi$ could be regarded as different states of the observer (or detector). A few words on the origin of our Lagrangian are in order: in the classical limit the first term in $\mathcal{L}$ corresponds to the Lagrangian for a inverted harmonic oscillator, that is

$$
L_{c l}=-m\left(\dot{z}^{2}+a^{2} z^{2}\right),
$$

and the sign is chosen so that the corresponding Hamiltonian is equal to the (positive) particle (detector) mass (the opposite choice is made in [5]); the second term describes the interaction between the detector and the scalar field $\varphi$ (whose Lagrangian is given by the last term). Further if one considers $Q=Q(\tau)$ as an operator acting on the Hilbert space of the detector's internal energy states, $a_{1}=a_{2}, m_{1}=m_{2}$ one needs only one field $\psi\left(=\psi_{1}=\psi_{2}\right)$ and our previous results are reproduced [4]. Instead we shall consider $Q$ a time independent c-number (coupling constant) and therefore the interaction with a quantum $\varphi$ is associated with the transition $\psi_{1} \rightarrow \psi_{2}$ corresponding to a change (which we shall always consider to be small) of acceleration and/or mass of the detector.

From the first term in $\mathcal{L}$ one obtains

$$
\begin{aligned}
\psi_{i}(z, \tau)= & \left(\frac{\beta_{i}}{i b \sqrt{\pi}}\right)^{1 / 2}\left(\frac{1}{2 b^{2}}-i \beta_{i} \cosh a_{i} \tau\right)^{-1 / 2} \\
& \times \exp \left\{i \beta_{i}\left[z^{2} \cosh a_{i} \tau+\frac{\alpha_{i}^{2}}{4 b^{2} \beta_{i}^{2}}\left(a_{i}^{-2} \cosh a_{i} \tau-2 z a_{i}^{-1}-4 z^{2} \beta_{i}^{2} b^{4} \cosh a_{i} \tau\right)\right]\right\} \\
& \times \exp \left\{-\frac{\alpha_{i}^{2}}{2}\left(z-a_{i}^{-1} \cosh a_{i} \tau\right)^{2}\right\},
\end{aligned}
$$

where $\beta_{i} \equiv-\frac{m_{i} a_{i}}{\hbar \sinh a_{i} \tau}$ and $\alpha_{i} \equiv \frac{2 b \beta_{i}}{\left(1+4 b^{4} \beta_{i}^{2} \cosh ^{2} a_{i} \tau\right)^{1 / 2}}$. This is a solution to the equation of motion (Schrödinger equation) with a gaussian wave-packet $\psi_{i}(z, 0)$ of width $b$ as initial condition. Since, as we have previously observed [4], the Unruh effect is obtained in the semiclassical limit $(\hbar \rightarrow 0$, $\beta_{i} \rightarrow-\infty$ and $b$ finite), it will be sufficient to use

$$
\psi_{i}(z, \tau) \simeq \frac{1}{\left(b \sqrt{\pi} \cosh a_{i} \tau\right)^{1 / 2}} \exp \left\{-\frac{i}{\hbar} m_{i} a_{i} z^{2} \tanh a_{i} \tau-\frac{\left(z-a_{i}^{-1} \cosh a_{i} \tau\right)^{2}}{2 b^{2} \cosh ^{2} a_{i} \tau}\right\} .
$$

The energy of the above wave function, Eq. (4), may be evaluated obtaining

$$
\left\langle H_{i}\right\rangle=m_{i}\left[\frac{\hbar^{2}}{4 m_{i}^{2}}\left\langle\psi_{i}\left|\frac{\partial^{2}}{\partial z^{2}}\right| \psi_{i}\right\rangle+a_{i}^{2}\left\langle\psi_{i}\left|z^{2}\right| \psi_{i}\right\rangle\right]=m_{i}
$$


which coincides with the Hamiltonian computed along the classical trajectory $z_{i}=a_{i}^{-1} \cosh a_{i} \tau$, agrees with the rest mass of the detector and occurs in the imaginary part of the exponent of $\psi_{i}$ for $\tau \rightarrow 0$ independently of the value of $a_{i}$.

Let us now consider the probability for the detector to make a transition from $a_{1}=a-\delta a / 2$, $m_{1}=m-\delta m / 2$ to $a_{2}=a+\delta a / 2, m_{2}=m+\delta m / 2$ associated with a quantum $\varphi$ of energy $|\delta m|$ $(\delta m<0$ for emission and $\delta m>0$ for absorption and we shall see later that $\delta a$ is related to $\delta m)$. On using the interaction Lagrangian density (second term in Eq. (1)) one obtains

$$
\begin{aligned}
P_{21}(\delta a, \delta m)= & \frac{Q^{2}}{4 \hbar^{2}} \int_{\tau_{1}}^{\tau_{2}} d \tau \int_{\tau_{1}}^{\tau_{2}} d \tau^{\prime} \int d z \int d z^{\prime} \psi_{1}^{*}\left(z^{\prime}, \tau^{\prime}\right) \psi_{2}\left(z^{\prime}, \tau^{\prime}\right) \psi_{1}(z, \tau) \psi_{2}^{*}(z, \tau) \\
& \times\left\langle 0\left|\varphi\left(z, x_{c}^{0}\right) \varphi\left(z^{\prime}, x_{c}^{0^{\prime}}\right)\right| 0\right\rangle \\
= & -\frac{Q^{2}}{4 \hbar^{2}} \int_{0}^{L} d\left(\tau-\tau_{1}\right) \int_{0}^{L} d\left(\tau^{\prime}-\tau_{1}^{\prime}\right) \int d z \int d z^{\prime} \psi_{1}^{\prime *} \psi_{2}^{\prime} \psi_{1} \psi_{2}^{\prime *} \\
& \times \frac{\hbar}{4 \pi} \ln \left[\left(z-z^{\prime}\right)^{2}-\left(x_{c}^{0}-x_{c}^{0^{\prime}}-i \epsilon\right)^{2}\right],
\end{aligned}
$$

where $x_{c}^{0}(\tau) \equiv\left(x_{1}^{0}(\tau)+x_{2}^{0}(\tau)\right) / 2, x_{c}^{0^{\prime}} \equiv x_{c}^{0}\left(\tau^{\prime}\right), \psi_{i}^{\prime} \equiv \psi_{i}\left(z^{\prime}, \tau^{\prime}\right)$. In evaluating $P_{21}$ it is convenient to examine the exponent of $\psi_{2}^{*} \psi_{1}$,

$$
\begin{aligned}
\ln \psi_{2}^{*} \psi_{1}= & -\frac{1}{2 b^{2}}\left[\frac{\left(z-a_{1}^{-1} \sinh a_{1} \tau\right)^{2}}{\cosh ^{2} a_{1} \tau}+\frac{\left(z-a_{2}^{-1} \sinh a_{2} \tau\right)^{2}}{\cosh ^{2} a_{2} \tau}\right] \\
& +\frac{i}{\hbar} z^{2}\left(m_{2} a_{2} \tanh a_{2} \tau-m_{1} a_{1} \tanh a_{1} \tau\right) \\
& -\frac{1}{2} \ln \left(b^{2} \pi \cosh a_{1} \tau \cosh a_{2} \tau\right) .
\end{aligned}
$$

On first considering the real part of the above it is immediate to see that $\left|\psi_{2}^{*} \psi_{1}\right|^{2}$ is peaked at $z_{c}=\left(a_{1}^{-1} \cosh a_{1} \tau+a_{2}^{-1} \cosh a_{2} \tau\right)$ up to $\mathcal{O}\left[(\delta a)^{2}\right]$ corrections. The imaginary part of Eq. (7) may then be evaluated at $z=z_{c}$, finally obtaining

$$
\frac{i}{2 \hbar a}\left[\left(\delta m+m \frac{\delta a}{a}\right) \sinh 2 a \tau+2 m \delta a \tau\right],
$$

again up to higher order corrections in $\delta a, \delta m$ and we have assumed $\delta a \tau$ is small (this will constrain the coupling constant - we shall return to this).

The above imaginary part of the exponent is associated with the change of energy between the final (2) and initial state (1), which, from Eq. (5), is expected to be $\delta m=m_{2}-m_{1}$. Hence requiring that Eq. (8) be equal to $-i \delta m \tau / \hbar$ leads to

$$
\delta m=-m \frac{\delta a}{a},
$$

or

$$
m=\frac{f}{a}
$$


where $f$ is a positive constant. Thus on demanding consistency (i.e., conservation of energy/momentum) we have obtained a relationship between mass and acceleration corresponding to the action of a constant force.

The above approximations and Eq. (9) may be substituted into Eq. (6) and on introducing $\tau=T+t / 2, \tau^{\prime}=T-t / 2, T^{\prime}=T-\tau_{1}$ one obtains

$$
\begin{aligned}
P_{21}(\delta a) & =-\frac{Q^{2}}{8 \pi \hbar} \int_{0}^{L} d T^{\prime} \int_{-L+2\left|T^{\prime}-L / 2\right|}^{+L-2\left|T^{\prime}-L / 2\right|} d t e^{i \frac{f \delta a}{\hbar a^{2}} t} \ln \left[\frac{2}{a} \sinh \left(\frac{a t}{2}-i \epsilon\right)\right] \\
& =-i \frac{Q^{2} a^{2}}{8 \pi f \delta a} \int_{0}^{L} d T^{\prime} \int_{-L^{\prime}}^{+L^{\prime}} d t \sum_{n=0}^{\infty} \frac{e^{i \frac{f \delta a}{\hbar a^{2}} t}}{\left(t-\frac{2 \pi i n}{a}-i \epsilon\right)},
\end{aligned}
$$

which is of the desired form (see, e.g., [6]) and we have omitted an end point contribution in the integration by parts [7]. One may evaluate the $t$ integral in Eq. (11) by closing the contour in the upper complex half plane $(\delta a>0)$, thus including the poles at $t=2 \pi i n / a$, with $n$ a non negative integer. One then obtains

$$
P_{21}(\delta a>0) \simeq \frac{Q^{2} a^{2} L}{4 f \delta a} \sum_{n=0}^{\infty} e^{-\frac{2 \pi f \delta a}{\hbar a^{3}} n},
$$

up to contour contributions associated with transient effects, the requirements for the neglect of which we shall return to afterwards. For the case $\delta a<0$ one performs the $t$ integration in Eq. (11) in the lower complex half plane. From the residues at $t=-2 \pi n i / a$ one obtains the same result as Eq. (12) with $\delta a$ replaced by $|\delta a|$ and the sum now runs from 1 to $\infty$ since the pole in $0+i \epsilon$, which is responsible of spontaneous emission/absorption, is excluded. Thus one finally obtains

$$
P_{21}(\delta a) \simeq \frac{Q^{2} a^{2} L}{4 f|\delta a|} \sum_{n}^{\infty} e^{-\frac{2 \pi f|\delta a|}{\hbar a^{3}} n}=\frac{Q^{2} a^{2} L}{4 f|\delta a|} \frac{\sigma(\delta a)}{1-e^{-\frac{2 \pi f \delta a}{\hbar a^{3}}}},
$$

where $\sigma(x)=+1$ for $x>0$ and $\sigma(x)=-1$ for $x<0$. In the above one notes the appearance of the familiar Planck distribution factor and the usual Unruh temperature $\beta^{-1}=\hbar a / 2 \pi$ (remembering that $\delta m=-f \delta a / a^{2}$ is the change in energy) with $a=\left(a_{1}+a_{2}\right) / 2$. Further we observe that for $f=1 / 4 G, \beta^{-1}=\hbar / 8 \pi G m$ which is the Hawking temperature for a black hole of mass $m$.

\section{Multiple emission/absorption and black hole analogy}

In the previous Section we have obtained an expression for the probability of emission (or absorption) of a scalar quantum by the accelerated observer with the corresponding back-reaction. In this section we illustrate a possible analogy with black holes.

To start with, let us note that for a generic accelerated observer one needs an external static source to produce the constant force $f$. Instead, if one identifies a black hole with its horizon (whose acceleration is equal to the surface gravity $f / m=1 / 4 G m$ and which is where Hawking emission takes place), the source of the force coincides with the black hole itself and thus one does not have an external source (as is the case in our model Eq. (1)). Hence, a change in the 
mass associated with the horizon is also a change in the strength (mass) $m$ of the source, while $f=1 / 4 G$ is constant (which is a statement of the equivalence principle).

One may proceed to calculate the change of energy (mass) per unit time [8] (see [9] for an analogous calculation for a black hole) for the accelerated observer emitting a "thermal" scalar field $\varphi$ (see Eq. (13)) due to the coupling in our Lagrangian (1). In particular the average loss of mass per unit time will then be given by

$$
\frac{\langle\delta m\rangle}{L} \propto-\frac{Q^{2}}{4} \int \frac{d \omega}{e^{\beta \hbar \omega}-1}=-\frac{Q^{2} f}{8 \pi m} \int \frac{d x}{e^{x}-1},
$$

where we have identified $\hbar \omega=f \delta a / a^{2}, x=\beta \hbar \omega$ and we shall not concern ourselves with eventual infrared divergencies which can be handled in the usual way [8]. Since we want the mass to decrease for increasing time (emission), corresponding to the Unruh vacuum for a black hole [6], on identifying (for $L$ sufficiently small) $\dot{m} \simeq\langle\delta m\rangle / L$ one obtains

$$
m(\tau)=m_{0}\left(1-\frac{\tau}{\tau_{d}}\right)^{1 / 2}
$$

which, on setting $f=1 / 4 G$,corresponds to the evaporation of a 2-dimensional black hole (in the Schwarzschild time $\tau$ ) with initial $(\tau=0)$ mass $m_{0}$ and decay time $\tau_{d} \propto 4 \pi m_{0}^{2} / f Q^{2}=$ $16 \pi G m_{0}^{2} / Q^{2}$. Correspondingly

$$
a(\tau)=\frac{f}{m_{0}}\left(1-\frac{\tau}{\tau_{d}}\right)^{-1 / 2} .
$$

On using Eq. (14) it is straightforward to see that $\beta\langle\delta m\rangle$ is constant. Clearly this result depends on the expression we obtained for $P_{21}$, in particular the presence of the $1 / \hbar \omega$ factor in Eq. (13) which in turn depends on the form of the interaction in Eq. (1).

The dependence of $a$ (or $m$ ) on time describes the semiclassical trajectory that the accelerated observer (black hole) follows. One may then imagine replacing the continuously changing acceleration $a(\tau)$ by a series of $N$ straight lines each associated with equal time intervals $L$ and the emission of a scalar quantum every time the line slope changes. More precisely one considers a trajectory beginning at $\tau_{0}=0$ for an accelerated detector having initial mass $m_{0}$ and ending with a final mass $m_{N}$ in a time interval $\tau_{N}-\tau_{0}=N L\left(\tau_{N} \leq \tau_{d}\right)$ after emitting $N$ quanta. Clearly $L$ must be sufficiently small so that one may reasonably approximate $a(\tau)$ in the above fashion and higher order terms be negligible.

According to the above one obtains for the probability of emission of $N$ quanta in the interval $N L$

$$
P_{N}=\prod_{r=1}^{N} P_{r, r-1} \simeq\left(\frac{Q^{2} L}{4}\right)^{N} \prod_{r=1}^{N} \frac{\left(\delta m_{r}\right)^{-1}}{e^{\beta_{r} \hbar \omega_{r}}-1}
$$

where of course $\delta m_{r}=m_{r}-m_{r-1} \equiv m(r L)-m((r-1) L)$ and $P_{r, r-1}$ is given by Eq. (13) with $a=\left(a_{r}+a_{r-1}\right) / 2$ and $\delta a=a_{r}-a_{r-1}$. A remarkable point is that with the time dependence for the trajectory given in Eq. (16) (evaporation) one has $\beta_{r} \hbar \omega_{r} \simeq \beta\langle\delta m\rangle=$ constant and obtains

$$
P_{N} \propto\left(\frac{4 \pi m_{0} / f}{e^{\beta\langle\delta m\rangle}-1}\right)^{N} \prod_{r=1}^{N}\left(1-\frac{r L}{\tau_{d}}\right)^{1 / 2}
$$


corresponding to a sequence of $N$ emissions at the most probable frequencies, that is the ones for which the exponents in the denominators of Eq. (17) are minimum.

Let us conclude by illustrating the constraints for the approximate validity of our approach. The final expression in Eq. (18) depends on the accelerating force $f$, the decay time $\tau_{d}$ (related to the coupling constant $Q$ ), the initial condition $m_{0}$ and the parameter $L$. The time interval $L$ is constrained by the requirements that $\delta a L \ll 1$ (see after Eq. (8)) and that the contour contributions in Eq. (11) are negligible, that is the exponent $f \delta a L / \hbar a^{2} \gg 1$. Thus one needs

$$
\frac{\hbar}{|\dot{m}|} \ll L^{2} \ll \frac{m^{2}}{f|\dot{m}|},
$$

which implies $m^{2} / f \gg \hbar$ or (for the black hole analogy $f=1 / 4 G$ ) $m \gg \sqrt{\hbar / G} \equiv m_{p}$, the Planck mass.

\section{References}

[1] S. W. Hawking, Nature 248 (1974) 30; Comm. Math. Phys. 43 (1975) 199.

[2] W. G. Unruh, Phys. Rev. D 14 (1976) 870.

[3] B. S. DeWitt in General Relativity: an Einstein Centenary Survey, S. W. Hawking and W. Israel eds. (Cambridge University Press, Cambridge, England, 1979).

[4] R. Casadio and G. Venturi, Phys. Lett. A 199 (1995) 33.

[5] R. Brout and Ph. Spindel, Nucl. Phys. B 348 (1991) 405; R. Brout, Z. Phys. B 68 (1987) 339.

[6] N. D. Birrell and P.C.W. Davies, Quantum fields in curved space (Cambridge University Press, Cambridge, England, 1982).

[7] S. Takagi, Prog. Theor. Phys., 74 (1985) 142.

[8] H. A. Weldon, Phys. Rev. D 49 (1994) 1579.

[9] D. N. Page, Phys. Rev. D 13 (1976) 198. 